\title{
Blick in die Zukunft pflanzlicher Arzneimittel
}

\author{
Beat Meier ${ }^{\mathrm{a}}$ Werner Knöss $^{\mathrm{b}} \quad$ Andreas Wenng $^{\mathrm{c}} \quad$ Reinhard Saller $^{\mathrm{d}}$ \\ ${ }^{a}$ Zürcher Hochschule für angewandte Wissenschaften, Fachgruppe Phytopharmazie, Wädenswil, Schweiz, \\ ${ }^{b}$ Bundesinstitut für Arzneimittel und Medizinprodukte (BfArM), Bonn, Deutschland, \\ 'Swissmedic, Schweizerisches Heilmittelinstitut, Abteilung Komplementär- und Phytoarzneimittel, Bern, \\ ${ }^{\mathrm{d}}$ Departement für Innere Medizin, Institut für Naturheilkunde, UniversitätsSpital Zürich, Schweiz
}

Eine ärztlich orientierte Phytotherapie ist darauf angewiesen, dass genügend pflanzliche Arzneimittel in bester Qualität zur Abgabe an den Patienten zur Verfügung stehen. In Deutschland, Österreich und der Schweiz steht seitens der Behörden die Infrastruktur für die Zulassung pflanzlicher Arzneimittel bereit, verbunden mit hohem sachlichem Know-how. Trotzdem ist ein deutlicher Trend zu beobachten: Zubereitungen aus Pflanzen mit therapeutischem Anspruch werden auch von renommierten Firmen in die Kosmetik oder zu den Nahrungsergänzungsmitteln ausgelagert sowie als Medizinprodukt gehandelt. Die regulatorischen Anforderungen sind deutlich geringer, Ideen können rascher umgesetzt und am Markt erprobt werden. Die Arbeit des Commitee on Herbal Medicinal Products (HMPC), das in den letzten Jahren 120 Monographien erarbeitet hat, wird demgegenüber nur zögerlich umgesetzt. Limitierend ist da die Dosisfrage, die in der Phytotherapie ungelöst bleibt. Es gibt nur einige wenige Beispiele, in denen mit Hilfe von Studien optimale Dosierungen von Extrakten ermit- telt wurden [1, 2], doch insgesamt sind die Bandbreiten sehr groß. Es ist nicht gesagt, dass über die Aufarbeitung der Daten zum «traditional use» die optimale Dosis gefunden wird. Als Beispiel sei Vitex-agnus castus, L. genannt: Die in Deutschland während Jahrzehnten gültige Dosierung, empfohlen durch die Kommission E, erwies sich im klinischen Versuch, der nach Hinweisen aus der Praxis durchgeführt wurde, als zu gering [3]. Entsprechend sollten die Dosierungen, die in den HMPC-Monographien empfohlen werden, nicht in Stein gemeißelt bleiben und flexibel gehandhabt werden. Die große therapeutische Breite der meisten pflanzlichen Zubereitungen ließe ein solches Vorgehen problemlos zu. Der Bedarf nach pragmatischer Forschung ist in der Phytotherapie nach wie vor enorm. Die Organisatoren der Tagung "Phytotherapie 2014» hoffen, dass diese einen Stimulus für die Zukunft setzen kann.

Beat Meier

\section{Literatur}

1 Schellenberg R, Saller R, Hess L, Melzer J, Zimmermann C, Drewe J, Zahner C: Dose-dependent effects of the Cimicifuga racemosa extract Ze450 in the treatment of climacteric complaints: a randomized placebo-controlled study. Evid Based Complement Alternat Med 2012;2012:260301.
2 Schapowal A: Butterbur Ze339 for the treatment of intermittent allergic rhinitis. Dose-dependent efficacy in a prospective, randomized, double-blind, placebo-controlled study. Arch Otolaryngol Head Neck Surg 2004;130:1383-1386.
3 Schellenberg R, Zimmermann C, Drewe J, Hoexter G. Zahner C: Dose-dependent efficacy of the Vitex agnus-castus extract Ze 440 in patients suffering from premenstrual syndrome. Phytomedicine 2012;19:1325-1331. 


\section{HMPC-Monographien - das Ende der Forschung in der Phytotherapie?}

\section{Autor: Werner Knöss}

Das Commitee on Herbal Medicinal Products (HMPC) besteht seit 10 Jahren - die Phytotherapie seit mehreren Tausend Jahren!

Der Ausschuss für pflanzliche Arzneimittel (HMPC) wurde im Jahre 2004 bei der Europäischen Arzneimittelagentur (European Medicines Agency (EMA), London) eingerichtet. Eine wesentliche Aufgabe war die Erstellung von Gemeinschaftsmonographien für pflanzliche Substanzen bzw. daraus hergestellte Zubereitungen. Diese Monographien sollten die Registrierung traditioneller pflanzlicher Arzneimittel und die Zulassung pflanzlicher Arzneimittel vereinfachen. Grundlage waren europäische Gesetzeswerke, die unter anderem das Ziel hatten, existierenden pflanzlichen Arzneimitteln den Verbleib im Markt zu ermöglichen, die Genehmigungsverfahren zu vereinfachen und die Bewertung pflanzlicher Arzneimittel zu harmonisieren [1-3].

Das HMPC hat zwischen 2004 und 2014 eine Vielzahl von Dokumenten und Leitlinien zur Bewertung der Qualität, Wirksamkeit und Unbedenklichkeit pflanzlicher Arzneimittel und traditioneller pflanzlicher Arzneimittel erarbeitet. Es wurden etwa 120 Gemeinschaftsmonographien zu pflanzlichen Substanzen und daraus hergestellten Zubereitungen verabschiedet. Der Prozess ist transparent und beinhaltet auch die Möglichkeit einer Kommentierung durch die Fach- und sonstigen interessierten Kreise. Leitlinien und Monographien sowie die dazu gehörenden Dokumente (Bewertungsbericht, Referenzliste, Übersicht über die Kommentare) stehen kostenfrei auf der Webseite der European Medicines Agency (EMA) zur Verfügung (www.ema.europa.eu). In etwa 20\% der Monographien des HMPC wurde ein so genannter «well-established» use festgestellt, in der Mehrzahl der Bewertungen konnte aufgrund der öffentlich verfügbaren Daten lediglich ein «traditional use» festgestellt werden. Die Mehrzahl der in der Europäischen Union in pflanzlichen Arzneimitteln gebräuchlichen pflanzlichen Substanzen wurde bereits in den Monographien des HMPC bewertet [4]. In den Mitgliedsstaaten wurden bereits mehr als 1000 Registrierungen für traditionelle pflanzliche Arzneimittel erteilt. Die gesetzlichen Regelungen wurden somit angemessen umgesetzt, auch wenn die Auswirkungen noch längst nicht abgeschlossen sind. Probleme der Klassifikation entstehen auch immer wieder durch Rechtsvorschriften für Lebensmittel und Medizinprodukte, die ebenfalls Regelungen für Produkte mit botanischem Ausgangsmaterial beinhalten [5].

Ziele der Gesetzgebung zur Errichtung des HMPC und zur Einführung eines vereinfachten Verfahrens zur Registrierung traditioneller pflanzlicher Arzneimittel waren unter anderem

- die Erhaltung der Vielfalt pflanzlicher Arzneimittel,

- $\quad$ eine harmonisierte Bewertung und
- im Verbund damit auch ein vereinfachter Zugang über nationale Grenzen hinweg.

Die Forschung wurde im Gesetz selbst nicht thematisiert. Warum also wird verschiedentlich die Hypothese aufgestellt, dass die HMPC-Monographien das Ende der Forschung in der Phytotherapie bedeuten? Es wird angeführt, dass die HMPC-Monographien einen Marktzugang ermöglichen, der weitgehend auf bestehenden Daten beruht und keine zusätzlichen Untersuchungen zur Wirksamkeit und Unbedenklichkeit erfordert. Das ist zutreffend. Somit kann ein pharmazeutischer Unternehmer einen Marktzugang erhalten, der für viele Jahre gültig sein kann und keine weitere Forschung erfordert. Auch diese Überlegung ist zutreffend. Allerdings ist dies nicht eine Folge der HMPC-Monographien, sondern zum Zeitpunkt der Gesetzgebung war bekannt, dass für eine Vielzahl von pflanzlichen Arzneimitteln die Voraussetzungen zum Beleg der Qualität, Wirksamkeit und Unbedenklichkeit für eine Zulassung nicht gegeben waren.

Es ist kaum anzunehmen, dass ohne neue gesetzliche Regelungen für zahlreiche pflanzliche Arzneimittel Forschungsprojekte etabliert worden wären. Dies erfolgte im Rahmen der sogenannten Nachzulassung in Deutschland für einige wenige Produkte. Die Mehrzahl der pflanzlichen Arzneimittel war entsprechend den Regelungen des deutschen Arzneimittelgesetzes [6] unter Bezug auf die Monographien der Kommission E zugelassen - ebenfalls ohne die Notwendigkeit aktueller Forschung.

Zumindest aus deutscher Sicht hat sich jedoch durch die Monographien des HMPC eine Änderung der Bewertung, ein neuer Stand der Erkenntnis ergeben. Für zahlreiche bislang zugelassene pflanzliche Arzneimittel ist entsprechend der Bewertung in den Monographien des HMPC bei neuen Entscheidungen für vergleichbare Wirkstoffe nur eine Registrierung vorgesehen. Das alleine motiviert noch keine Forschung. Jedoch ist es vielleicht auch lohnenswert, ein wenig vorauszuschauen: In einer Welt der Globalisierung und des stetig, wenn nicht gar exponentiell wachsenden Wissens kann in nicht allzu ferner Zukunft von den Patientinnen und Patienten die Frage gestellt werden, welche wissenschaftliche Grundlage für die Anwendung eines Arzneimittels besteht. Möchte ein pharmazeutisches Unternehmen nicht für kurze Zeiträume planen sondern für die Zukunft, so ergibt sich zwangsläufig die Notwendigkeit, in Forschung zu investieren. Ein Überblick über wissenschaftliche Zeitschriften verdeutlicht, dass bereits die Mehrzahl von Publikationen zu Arzneipflanzen und deren Zubereitungen von asiatischen Autorenteams eingebracht wird. Neue analytische Techniken und Untersuchungsmethoden eröffnen Möglichkeiten, in Forschungsprojekten pflanzliche Arzneimittel und ihre Wirkungen zu charakterisieren. Auch die klinische Relevanz einer Therapie kann mit epidemiologischen Methoden analysiert werden. Die HMPC-Monographien waren nicht als Instrument einer Forschungsförderung gedacht, aber aktuell haben sie zum Erhalt pflanzlicher und traditioneller pflanzlicher Arzneimittel in Europa beigetragen und stellen bestehende Wissenslücken fest. Auf lange Sicht ge- 
sehen können sie auch ein Ausgangspunkt für weitere Forschung in der Phytotherapie sein.

\section{Literatur}

1 Richtlinie 2001/83/EG des Europäischen Parlaments und des Rates vom 06. November 2001 zur Schaffung eines Gemeinschaftskodexes für Humanarzneimittel (ABl. L 311 vom 28. November 2001) und nachfolgende Änderungen.

2 Verordnung (EG) Nr. 726/2004 des Europäischen Parlaments und des Rates vom 31. März 2004 zur Festlegung von Gemeinschaftsverfahren für die Genehmigung und Überwachung von Human- und Tierarzneimitteln und zur Errichtung einer Europäischen Arzneimittel-Agentur (ABl. L 136 vom 30.04.2004, S. 1).

3 Richtlinie 2004/24/EG des Europäischen Parlaments und des Rates vom 31. März 2004 zur Schaffung eines Gemeinschaftskodexes für Humanarzneimittel hinsichtlich traditioneller pflanzlicher Arzneimittel (ABl. L 136 vom 30.04.2004, S. $85)$.

4 Knöss W, Chinou I: Regulation of medicinal plants for public health - European community monographs on herbal substances. Planta Medica 2012;78:1-6.

5 Stephan K: Wie sind Arzneimittel von anderen Gesundheitsprodukten abzugrenzen? Pharmazie in unserer Zeit 2011;40:300-304.

6 Arzneimittelgesetz in der Fassung der Bekanntmachung vom 12. Dezember 2005 (BGBl. I S. 3394), das zuletzt durch Artikel 1 des Gesetzes vom 10. Oktober 2013 (BGBl. I S. 3813) geändert worden ist.

\section{Kommt für die Phytotherapie der Weg zurück zum Arzneimittel?}

\section{Autor: Andreas Wenng}

Im Jahr 2013 waren bei Swissmedic 655 phytotherapeutische Arzneimittel zugelassen. Im Jahr 2011 waren es 739 und 2012734 [1]. Bricht man das auf die in den zugelassenen Präparaten verwendeten Arzneipflanzen herunter, sind das ungefähr 115. Bei 850 in der Schweiz vorkommenden dokumentierten Arzneipflanzen ist damit der Bestand an pflanzlichen Arzneimitteln eher gering.

Jährlich kommen aktuell nur ca. 1-3 pflanzliche Arzneimittel mit neuen Wirkstoffen hinzu. 2013 war es 1 Phytoarzneimittel. Auch die Zahl neu zugelassener Phytoarzneimittel mit bekannten Wirkstoffen ist überschaubar.

Für diese schwache Entwicklung sind mehrere Gründe anzuführen, die aber nur zu einem geringen Maß an der Zulassungspraxis der Swissmedic liegen. Auszuschließen sind die Zulassungskosten, die für ein pflanzliches Arzneimitteln mit neuem Wirkstoff nach der Gebührenverordnung [2] bei 6000 CHF, bei bekannten Wirkstoffen bei 3000 CHF liegen. Zudem gibt es für die meisten Phytotherapeutika vereinfachte Zulassungsverfahren, sofern die entsprechenden Voraussetzungen erfüllt sind. Diese sind in der Komplementär- und Phytoarzneimittelverordnung (KPAV) [3] und weitergehend in der Phytoanleitung [4], einer Verwaltungsverordnung, geregelt.

Weit größeren Einfluss haben die Kosten, die für einen evidenzbasierten Wirksamkeitsnachweis erbracht werden müssen. Dies hält wohl viele Unternehmen davon ab, für neue pflanzliche Arzneimittel den Schritt der Zulassung zu gehen.
Ein weiterer Punkt ist die zweite Revision des Heilmittelgesetzes [5] in der Schweiz. Das revidierte Gesetz tritt voraussichtlich 2017 in Kraft. Die laufenden inhaltlichen Diskussionen könnte die Industrie momentan abwarten lassen.

Nicht beeinflussbar durch die Behörde ist der Markt selbst und die Verordnungs- bzw. Verschreibungshäufigkeit pflanzlicher Arzneimittel durch den Arzt. Auch auf die Vergütung durch die Grundversicherung hat die Swissmedic keinen Einfluss.

Seit einiger Zeit sind zunehmend Trends zu beobachten, pflanzliche Präparate über andere Schienen als den Arzneimittelsektor auf den Markt zu bringen: Einige Pflanzen, die früher Wirkstoffe in zugelassenen Arzneimitteln waren, finden sich heute ganz legal in Medizinprodukten. Da die Schweiz im europäischen Verbund die europäischen Regelungen für Medizinprodukte anerkennt und für diese Produkte ein freier Warenverkehr in Europa besteht, sind Swissmedic hier die Hände gebunden. Und es ist schwierig, diesen Markt zu kontrollieren. Auch Nahrungsergänzungsmittel und Kosmetika sind Produktgruppen, in denen viele Hersteller ihre neuen Produkte positionieren.

Hier gilt es, die Vorteile einer Zulassung als Arzneimittel sowohl bei den Herstellern als auch in der Öffentlichkeit besser bekannt zu machen. Letztendlich dient der enorme Aufwand dem Patienten. Ein zugelassenes Arzneimittel ist eine Garantie für Qualität, Sicherheit und Wirksamkeit. Die «Abwanderungen» in andere Bereiche zu beobachten und wo nötig auch einen «Graumarkt» zu unterbinden ist eine der größten Herausforderungen einer Behörde.

Auch bei der Zulassung selbst hat Swissmedic mit ihrer Abteilung KPA einige Möglichkeiten zur Weichenstellung in Richtung Förderung der Phytotherapie.

Ein Schritt ist eine intensivere Diskussion mit den europäischen Behörden, vornehmlich mit Deutschland und Österreich, um sich besser abzustimmen. Eine solche Zusammenarbeit ist seit Beginn 2014 mit dem Bundesinstitut für Arzneimittel und Medizinprodukte (BfArM) geregelt.

Weitere Schritte liegen innerhalb der Zulassungsprozesse:

Swissmedic bietet mit den Instrumenten «clarification meeting» und «scientific advice» schon in der Frühphase einer $\mathrm{Zu}$ lassung dem Gesuchsteller Hilfestellung. Dieser Art der Begleitung wurde bisher zu wenig Beachtung geschenkt. Der Wandel der Behörde zum Dienstleister innerhalb des vorgegebenen gesetzlichen Rahmens, ohne das Mandat der Gleichbehandlung zu verletzen, hat gerade erst begonnen. Auch jungen «start ups» kann man so eine Einstiegshürde nehmen.

Eine Vereinfachung des Einreichungsverfahrens liegt darin, zukünftig weitgehend auf Papier zu verzichten - Stichwort: e-Portal. Aber dies geht nur reibungslos, wenn die Daten des Einreichers eine gute Qualität haben. Weiterhin hat der Antragsteller zukünftig im Rahmen des e-Portals die Möglichkeit, den Stand seines Gesuches abzurufen.

Grundsätzlich wird auch Artikel 13 des Heilmittelgesetzes zu wenig genutzt. Dieser besagt, dass, wenn ein Arzneimittel 
bereits in einem anderen Land mit vergleichbarer Arzneimittelkontrolle zugelassen ist, die Ergebnisse der dafür durchgeführten Prüfungen berücksichtigt werden. Im Jahr 2013 wurde nur auf ein Gesuch eingegangen.

Bei der Begutachtung innerhalb von Swissmedic kann in vielen Fällen gemäß einem «risk-based approach» die Tiefe der Begutachtung präparatespezifisch reduziert werden. Im Zentrum steht die Risiko-Nutzen-Abwägung.

Das alles reicht allerdings bei weitem nicht. Es muss intensiver geforscht werden. Neue Indikationen müssen erschlossen werden, und es bedarf einer Aufbruchstimmung, diese wertvollen pflanzlichen Präparate marktfähig zu halten.

\section{Literatur}

1 Swissmedic: Jahresberichte 2011 und 2012.

2 Swissmedic: Verordnung über die Gebühren des Schweizerischen Heilmittelinstitutes vom 1. Oktober 2006

3 Schweizerische Eidgenossenschaft: Verordnung des Schweizerischen Heilmittelinstituts über die vereinfachte Zulassung von Komplementär- und Phytoarzneimitteln vom vom 22. Juni 2006 (Stand 1. Juni 2011). 812.212.24.

4 Swissmedic: Anleitung zum Einreichen von Zulassungsgesuchen für pflanzliche Arzneimittel der Humanmedizin (Phyto-Anleitung) vom 1. Oktober 2006

5 Schweizerische Eidgenossenschaft: Bundesgesetz über Arzneimittel und Medizinprodukte (Heilmittelgesetz, HMG) vom 15. Dezember 2000 (Stand 1. Januar 2014). 812.21

\section{Die Dosisfrage in der Phytotherapie}

\section{Autor: Reinhard Saller}

Phytotherapeutika besitzen komplexe Wirkstoffe mit Vielstoffcharakter. Diese weisen genuin pleiotrope und Multi-target-Eigenschaften auf (Möglichkeit einer Polypharmakologie mit einem einzelnen Wirkstoff). Dementsprechend lassen sich zahlreiche Phytotherapeutika vielfältig und in mehreren Anwendungssituationen therapeutisch einsetzen. Wegen ihres wirkstoffbedingt breiten Wirkungsspektrums könnten sie sich auch in komplexen Therapiesituationen (z.B. Multimorbidität) als geeigneter Therapiebestandteil erweisen, gewissermaßen als polypharmakotherapeutischer Ansatz mit einem einzigen Arzneimittel [1]. Der Multi-Komponenten-Wirkstoff ist in pharmakologischer und vor allem therapeutischer Hinsicht nur bedingt über die stoffliche Analyse seiner Bestandteile hinreichend charakterisiert; es müssen funktionelle Gesichtspunkte mit herangezogen werden, d.h. das Wirkungsgefüge, das sich aus den vielfältigen Effekten der zumeist in niedriger Konzentration vorhandenen Komponenten und deren Interaktionsmöglichkeiten innerhalb des Wirkstoffes und mit den möglichen «targets» des behandelten Organismus (Netzwerkmodell pflanzlicher Arzneimittel, Plastizität des Wirkstoffgefüges) ergibt. Dass funktionell orientierte Betrachtungen unmittelbar therapeutisch relevant sein können, zeigen die verschiedenen Johanniskrautextrakte, die trotz quantitativ und teils auch qualitativ unterschiedlicher stofflicher Zusammensetzung bei adäquater Dosierung eine vergleichbare antidepressive Wirksamkeit zeigen.

Auch wenn die pflanzlichen Wirkstoffe sehr komplex sind, so ist selbstverständlich davon auszugehen, dass auch bei ihnen Dosis-Wirksamkeits-Beziehungen gelten. Allerdings wurden solche Beziehungen bislang nur selten systematisch untersucht, dabei vor allem bei modernen und patentierbaren Extrakten. Die meisten klinischen Studien zu Phytotherapeutika sind derzeit Untersuchungen mit nur einer Dosierung. Dosisabhängigkeit der jeweils indikationsbezogenen therapeutischen Wirksamkeit bzw. indikationsbezogene partielle Dosis-Wirkungs-Kurven wurden bislang nur selten geprüft, z.B. für einen Ginkgo-biloba-Extrakt [2], ein Ginkgo-biloba-haltiges Kombinationspräparat [3], einen Piper-methysticum-Extrakt [4], Rosmarinpulver [5], Zubereitungen aus Atropa belladonna [6], einen Valeriana-officinalis-Extrakt [7], für ein Tanacetum-parthenium- [8] und Melissa-officinalis-Präparat [9], einen Crataegus-Extrakt [10], einen Pelargonium-sidoides-Extrakt [11,12], einen Blattextrakt aus Petasites hybridus [13] sowie eine Zubereitung aus Astralagus [14], einer chinesischen Arzneidroge, die zunehmend in der westlichen Phytotherapie verwendet wird. Die Wirksamkeit nahm dabei jeweils dosisabhängig zu. Auch für Johanniskrautextrakte liegen klinische Studien vor, die im Rahmen einer individuell möglichen Dosissteigerung auf eine dosisabhängige antidepressive Wirksamkeit hinweisen $[15,16]$. Vergleichbar zeigt sich auch das klinische Interaktionspotenial dosisabhängig [17].

Häufig weist eine reflektierte therapeutische Erfahrung darauf hin, dass Dosierungen von Phytotherapeutika, wie sie in den Zulassungen festgelegt sind, für eine Reihe von Patienten nicht ausreichend sind und der zugelassene Dosisrahmen zu eng gefasst ist. Klinische Studien zeigen dies etwa am Beispiel eines Vitex-agnus- [18] und eines Cimicifuga-Extraktes [19]. Beide Studien über Dosis-Wirkung-Beziehungen belegen die indikationsbezogene dosisabhängige Zunahme der therapeutischen Wirksamkeit (prämenstruelles Syndrom bzw. postmenopausale Symptomatik) deutlich über den bisherigen Dosisrahmen der Zulassung hinaus. Häufigkeit und Schwere unerwünschter Wirkungen nahmen nicht zu.

Die Gründe für die relativ häufig (zu) niedrigen Dosierungsempfehlungen, die das therapeutische Potential von Phytotherapeutika nicht ausschöpfen bzw. eine Reihe von Patienten nicht hinreichend behandeln lassen, scheinen vielschichtig zu sein. Eine wesentliche Ursache könnte die direkte Übernahme der Dosisempfehlungen einschlägiger Drogen-Monografien sein. Der dortige Dosierungsrahmen erscheint häufig als eine formale, wissenschaftliche, therapeutische und regulatorische Kompromissbildung, die verständlicherweise eher niedrige und vor allem als «sicher» angesehene Dosierungsbereiche zu bevorzugen scheint. Wenn sich die Therapieforschung nur in solchen Dosierungsrahmen bewegt, können Forschungsergebnisse leicht zu selbsterfüllenden Prophezeiungen werden. So hat z.B. die Dosierungsempfehlung der Kommissi- 
on E bzw. der WHO-Monografie für die innerliche Anwendung von Johanniskraut («Soweit nicht anders verordnet: mittlere Tagesdosis 2-4 g Droge» bzw. «daily dosage: $2-4$ g crude drug») über lange Zeit klinische Forschung und Anwendung von Johanniskrautzubereitungen als Antidepressiva bestimmt und diesen Dosisrahmen quasi fixiert. Die empfohlenen Dosierungen wurden nahezu regelhaft auch als Rohstoffmengen für die Extraktherstellung verwendet. Bislang wurde kaum darüber hinaus gegangen, obwohl Forschungsergebnisse aus der Zeit nach der Monografieerstellung und vor allem die therapeutische Empirie dafür sprechen. Die HMPC/EMA-Monografie für die traditionelle Anwendung erweitert den Rahmen auf 3-6 g Droge/Tag.

Derzeit lässt sich nur sehr vorsichtig abschätzen, wie steil diese Dosis-Wirkung-Beziehungen verlaufen könnten, da zumeist nur 2 Dosisstufen geprüft wurden. Die bisherigen Daten und vor allem die therapeutische Empirie legen es nahe, von zumeist relativ flach verlaufenden Dosis-Wirkung-Beziehungen auszugehen. Dementsprechend könnten für eine relevante Steigerung der Wirksamkeit häufig eher größere Dosiserhöhungen sinnvoll sein. Wegen der bisherigen weitgehenden Dominanz von klinischen Studien mit nur einer phytotherapeutischen Dosierung lässt sich derzeit das Plateau der Dosis-Wirksamkeit-Beziehungen (Maß für eine maximal erzielbare Wirksamkeit) nicht abschätzen oder nur individualempirisch austesten. Möglicherweise wird dadurch das Potential einer Reihe von Phytotherapeutika unterschätzt.

Aus wissenschaftlichen und insbesondere therapeutischen Gründen müssten die in Monografien festgelegten Dosierungen wesentlich flexibler gehandhabt werden können. Wissenschaftlich bearbeitet werden müsste die Frage, ob und inwieweit Daten zur Dosierung zwischen mehr oder minder unterschiedlichen Extrakten übertragbar sein könnten.

$\mathrm{Zu}$ einem sinnvollen Umgang mit dieser Dosisproblematik könnten Forschungsansätze der pragmatischen Forschung und der Versorgungsforschung beitragen, sofern entsprechende Studien nicht nur den Status quo überkommener Dosierungen widerspiegeln. Ebenso könnten entsprechende qualifizierte Fallberichte außerordentlich nützlich sein.

Die Dosisfrage ist zum Teil bei einfachen phytotherapeutischen Zubereitungen noch komplexer. Bei Tees bezieht sich Dosierung nicht nur auf die eingesetzte Drogenmenge sondern unter anderem auch auf die Zeit, die man einen Tee ziehen lässt. Hinzu kommt, dass sich abhängig von der Zeit zum Ziehenlassen auch der Wirkstoff quantitativ und qualitativ ändern kann. Derzeit werden von vielen Patienten eher kürzere Extraktionszeiten als die tradierten Empfehlungen (5-10$15 \mathrm{~min}$ ) bevorzugt. Außerdem können sensorische Wahrnehmungen (Geruch, Geschmack) die Wirksamkeit bedeutsam beeinflussen und mögliche Dosis-Wirkung-Beziehungen nach systemischer Resorption modifizieren. Sowohl bei der Dosierung von Tees wie auch von Tinkturen spielt derzeit Tradition und Individualempirie eine entscheidende Rolle. Die Erfah- rungen weisen auf einen relativ großen interindividuell variablen Dosierungsrahmen hin.

Neben der jeweiligen indikationsbezogenen Wirksamkeit besitzen die phytotherapeutischen Wirkstoffe aufgrund ihres Vielstoff- und Netzwerkcharakters weitere potentiell therapeutisch relevante und dosisabhängige Wirkungen. Die entsprechenden Dosierungsfragen stellen eine weitere komplexe und reizvolle wissenschaftliche sowie regulatorische Herausforderung dar.

\section{Literatur}

1 Saller R, Rostock M: Multimorbidität und Multi-Target-Therapie in der Phytotherapie. Praxis 2012;101:1637-1642.

2 Woelk H, Arnoldt KH, Kieser M, Hoerr R: Ginkgo biloba special extract EGb 761 in generalized anxiety disorder and adjustment disorder with anxious mood: a randomized, double-blind, placebo-controlled trial. Psychiat Res 2007, 41:472480.

3 Scholey AB, Kennedy DO: Acute, dose-dependent cognitive effects of Ginkgo biloba, Panax ginseng and their combination in healthy young volunteers: differential interactions with cognitive demand. Hum Psychopharm 2002;17:35-44.

4 Sarris J, Stough C, Teschke R, Wahid ZT, Bousman CA, Murray G, Savage KM, Mouatt P, Ng C, Schweitzer I: Kava for the treatment of generalized anxiety disorder RCT: analysis of adverse reactions, liver function, addiction, and sexual effects. Phytother Res 2013;27:1723-1728.

5 Pengelly A, Snow J, Mills SY, Scholey A, Wesnes K, Butler LR: Short-term study on the effects of rosemary on cognitive function in an elderly population. J Med Food 2012;15:10-17.

6 Bettermann H, Cysarz D, Portsteffen A, Kummell HC: Bimodal dose-dependent effect on autonomic, cardiac control after oral administration of Atropa belladonna. Auton Neurosci 2001;90:132-137.

7 Gutierrez S, Ang-Lee MK, Walker DJ, Zacny JP:Assessing subjective and psychomotor effects of the herbal medication valerian in healthy volunteers. Pharmacol Biochem Behav 2004;78:57-64.

8 Pfaffenrath V, Diener HC, Fischer M, Friede M, Henneicke-von Zepelin HH, Investigators: The efficacy and safety of Tanacetum parthenium (feverfew) in migraine prophylaxis - a double-blind, multicentre, randomized placebo-controlled dose-response study. Cephalalgia 2002;22:523-532.

9 Kennedy DO, Wake G, Savelev S, Tildesley NT, Perry EK, Wesnes KA, Scholey $\mathrm{AB}$ : Modulation of mood and cognitive performance following acute administration of single doses of Melissa officinalis (Lemon balm) with human CNS nicotinic and muscarinic receptor-binding properties. Neuropsychopharmacology 2003:28:1871-1881.

10 Tauchert M: Efficacy and safety of crataegus extract WS 1442 in comparison with placebo in patients with chronic stable New York Heart Association class-III heart failure. Am Heart J 2002;143:910-915.

11 Matthys H, Lizogub VG, Malek FA, Kieser M: Efficacy and tolerability of EPs 7630 tablets in patients with acute bronchitis: a randomised, double-blind, placebo-controlled dose-finding study with a herbal drug preparation from Pelargonium sidoides. Curr Med Res Opin 2010;26:1413-1422.

12 Kamin W, Maydannik VG, Malek FA, Kieser M: Efficacy and tolerability of EPs 7630 in patients (aged 6-18 years old) with acute bronchitis. Acta Paediatri 2010;99:537-543.

13 Schapowal A, Petasites Study G: Butterbur Ze339 for the treatment of intermittent allergic rhinitis: dose-dependent efficacy in a prospective, randomized, double-blind, placebo-controlled study. Archi Otolaryngol 2004;130:1381-1386.

14 Yang QY, Lu S, Sun HR: Clinical effect of Astragalus granule of different dosages on quality of life in patients with chronic heart failure. Chin J Integr Med 2011;17:146-149.

15 Szegedi A, Kohnen R, Dienel A, Kieser M: Acute treatment of moderate to severe depression with hypericum extract WS 5570 (St John's wort): randomised controlled double blind non-inferiority trial versus paroxetine. BMJ 2005;330:503

16 Kasper S, Anghelescu IG, Szegedi A, Dienel A, Kieser M: Superior efficacy of St John's wort extract WS 5570 compared to placebo in patients with major depression: a randomized, double-blind, placebo-controlled, multi-center trial. BMC Med 2006;4:14. 
17 Mueller SC, Uehleke B, Woehling H, Petzsch M, Majcher-Peszynska J, Hehl EM, Sievers H, Frank B, Riethling AK, Drewelow B: Effect of St John's wort dose and preparations on the pharmacokinetics of digoxin. Clini Pharm Ther 2004;75:546557.

18 Schellenberg R, Zimmermann C, Drewe J, Hoexter G, Zahner C: Dose-dependent efficacy of the Vitex agnus castus extract Ze 440 in patients suffering from premenstrual syndrome. Phytomedicine 2012;19:1325-1331.

19 Schellenberg R, Saller R, Hess L, Melzer J, Zimmermann C, Drewe J, Zahner C: Dose-dependent effects of the cimicifuga racemosa extract Ze 450 in the treatment of climacteric complaints: a randomized, placebo-controlled study. Evid Based Complement Alternat Med 2012;2012:260-301

\section{Disclosure Statement}

Beat Meier: Die Fachgruppe Phytopharmazie an der ZHAW wird von Firmen und Verbänden finanziell unterstützt. Diese Verbindungen sind auf der Homepage der Fachgruppe offengelegt: http://phytotherapie2014. smgp.ch.

Werner Knöss: Es besteht kein Interessenkonflikt. Die Inhalte dieses Artikels und der Präsentation geben die persönliche Sicht des Autors wieder. Die dargestellten Inhalte sind weder aufzufassen noch zu zitieren als Positionen des HMPC (EMA) oder des BfArM.

Andreas Wenng: Es besteht kein Interessenkonflikt.

Reinhard Saller: Es besteht kein Interessenkonflikt. 